Old Dominion University

ODU Digital Commons

Electrical \& Computer Engineering Faculty

Publications

Electrical \& Computer Engineering

1998

Modeling Electromagnetic Disturbances in Closed-Loop

Computer Controlled Flight Systems

W. Steven Gray

Oscar R. Gonzalez

Follow this and additional works at: https://digitalcommons.odu.edu/ece_fac_pubs

Part of the Controls and Control Theory Commons, and the Electromagnetics and Photonics Commons 


\title{
Modeling Electromagnetic Disturbances in Closed-Loop Computer Controlled Flight Systems
}

\author{
W. Steven Gray Oscar González \\ Department of Electrical and Computer Engineering \\ Old Dominion University \\ Norfolk, VA 23529-0246
}

gray@ece.odu.edu gonzalez@ece.odu.edu

\begin{abstract}
High intensity electromagnetic radiation has been demonstrated to be a source of computer upsets in commercially available digital flight control systems. In this paper we introduce an electromagnetic disturbance model which can be used for stability analysis and augmentation of any such digitally implemented control law. The model is composed of a Markovian exosystem supplying radiation events to a discretetime jump linear system which models how the radiation interferes with the nominal operation of the closed-loop system. We discuss how this model can be used to characterize stability and how it can be parameterized and validated in an experimental setting.
\end{abstract}

\section{Introduction}

With the introduction of fly-by-wire systems in civilian aviation, the problem of designing, implementing, testing and certifying highly reliable computer control systems has become a major challenge to the industry and the FAA $[5,16]$. Aside from the usual problems of plant uncertainty, sensor and actuator failures, and environmental uncertainties, avionic systems are also subject to electromagnetic disturbances from both natural and man-made sources [11]. Such electromagnetic disturbances can introduce transient signals on analog sensor and actuator lines, change data values on digital input-output buses, or even produce logic changes in the CPU (see $[2,3,11]$ and the references therein). The result of these so called computer upsets is the introduction of some degree of degradation in the quality of the control signal ranging from a perturbation error over a few sample periods to a permanent error mode or computer failure. While the area of fault tolerant computing is a well established field of study, the classical focus has been on either compensating for sensor and actuator failures, detecting and/or correcting for abrupt changes in the plant due to subsystem failure or developing methods for incorporating redundancy to protect against wide scale computer failure $[6,7,15]$. In the worst case scenario, electromagnetic disturbances are capable of introducing all three types of failures, but the more likely situation is where intermittent or transient errors are introduced into the control calculation. While they may not cause an immediate failure of the system, these disturbances still can have some aggregate effect on closed-loop stability. This problem has received significantly less attention theoretically and experimentally, but it is no less important in ensuring controller reliability.

The main approaches addressing this problem to date fall into three general categories: improved shielding for the sensor/actuator lines and flight computers, control law enhancement, and fault detection (for isolation and correction). The latter two approaches are currently in an analysis phase; design methodologies are largely nonexistent or ad hoc. In this paper we take a first step toward developing a design methodology by considering how to model an electromagnetic disturbance with the specific intent of using this model for stability augmentation. Implicit in the approach taken here is the assumption that the upset condition is mild enough to prevent the system from going into a permanent error mode. Hence, the error in the control signal as a result of electromagnetic interference might be modeled as a structural perturbation of the ideal control law, an additive noise disturbance to the ideally computed control law, or perhaps some combination of both. The most comprehensive approach to this problem appears in $[8,14]$. (A somewhat related continuoustime problem appears in [12].) In this work the upset/non upset condition is modeled using a Markovchain derived by assuming that the arrival times of the interference have a Poisson distribution and the durations of each disturbance have an exponential distribution. (The probability of two events overlapping is tacitly taken to be zero.) When the upset state is entered, a stochastic perturbation to the control law is introduced into the closed-loop system. The aggregate model becomes a homogeneous stochastic linear difference equation for which the mean dynamics are computed and a stability analysis is performed.

Using a Markov-chain to switch between a set of 
linear closed-loop models is not a new concept in fault tolerant computing. These so-called jump linear systems and their stability properties have been studied by several researchers (see, for example, $[4,9,12,13]$ ). An obvious problem in using these models is deriving estimates for and/or validating the state and transition probabilities, especially if these parameters are both environment and system dependent. An even more difficult problem for this application is determining a model that accurately reflects the way the disturbance interferes with the normal operation of the system, i.e., the nature of the perturbation to the control signal. The complexity of the typical flight control system makes it impossible to derive such a model from physical principles. Furthermore, even if it were possible, all models would be hardware and software dependent. Changing a line of code in the flight computer could theoretically change its electromagnetic susceptibility.

In this paper we introduce an electromagnetic disturbance model which is also based on a Markovian process, but with several fundamental differences over what has been done before:

1. Our primary concern is in developing a model that can be parameterized and validated using only experimental data from an actual flight control computer system. (The authors have experimental access to a fault tolerant flight controller mounted in an electromagnetic test chamber developed by the HIRF Laboratory at the NASA Langley Research Center in Hampton, Virginia[2].)

2. The disturbance, when present, will be modeled by a noise injection into the closed-loop system and/or a deterministic perturbation to the digitally implemented control law.

3. The probability of two or more disturbances being present during a given sample period is not necessarily zero.

As will be explained in later sections, these innovations are somewhat interrelated.

The paper is organized as follows. In Section 2, we first introduce the basic structure of our disturbance model. Then in two subsections we outline the basic theory behind each of the two main components of the model: the exosystem and the interference model. In the final section we summarize our findings and propose future research.

\section{The Disturbance Model}

In general there are two basic parts in any disturbance model for a control problem: a model for the exosystem (any part of the system which is not the plant, sensors, controller or actuators) and an interference model which describes exactly how the exosystem interferes with the normal operation of the closed-loop system. Motivated by the work in [8], we examine two Markovian models for the exosystem

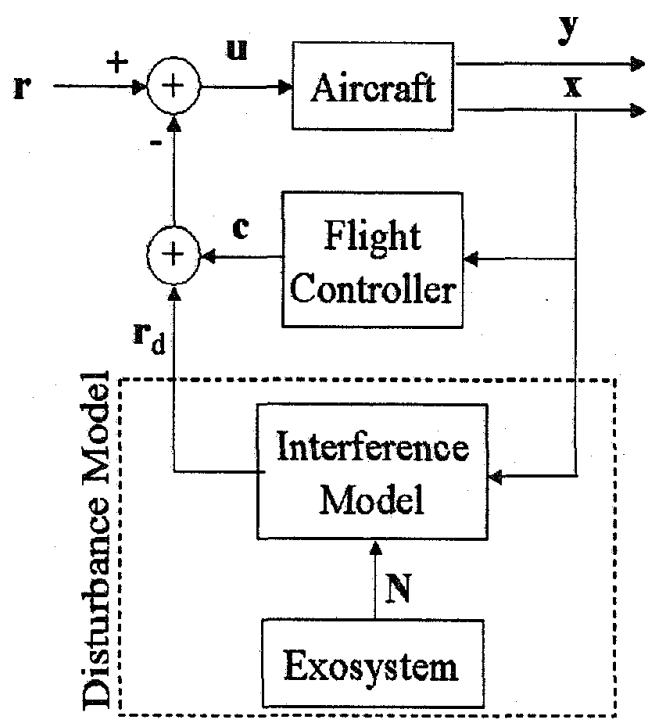

Figure 1: A typical closed-loop flight control system with the proposed electromagnetic disturbance model

and relate them to classic queuing models. We then show that the model in [8] is simply a special case of one of these queuing models. The proposed interference model consists of a signal injection system and/or a control law perturbation, predicated on the fact that all model parameters must be derived from input-output data taken from an electromagnetic test chamber. The overall system is represented in Figure 1. Each part of the disturbance model is now considered in more detail below.

\section{The Exosystem}

The typical flight control computer operates in a complex electromagnetic environment consisting of radiation at many different frequencies, powers, and angles of incidence. A drastically simplified model for this environment is to enumerate the number of active electromagnetic disturbances at any specific time instance $t \in \mathbb{R}$, call this integer $N(t)$. In this model, the $i$-th disturbance is characterized by its arrival time, $t_{i}$, and its total duration, $d_{i}$. We make the following initial assumptions concerning the sources and nature of these disturbances:

(A1) The random variables $t_{i}$ are defined by a Poisson process with constant parameter $\lambda$.

(A2) The random variables $d_{i}$ have an exponential distribution with parameter $\mu$.

(A3) The process $\mathbf{N}(t)$ is assumed to be a memoryless (Markovian) continuous-time random process, that is, for any small $\Delta t>0$ it follows that

$$
\begin{aligned}
P\{\mathbf{N}(t+\Delta t) \leq & k \mid \mathbf{N}(\tau), \tau \leq t\} \approx \\
& P\{\mathbf{N}(t+\Delta t) \leq k \mid \mathbf{N}(t)\} .
\end{aligned}
$$




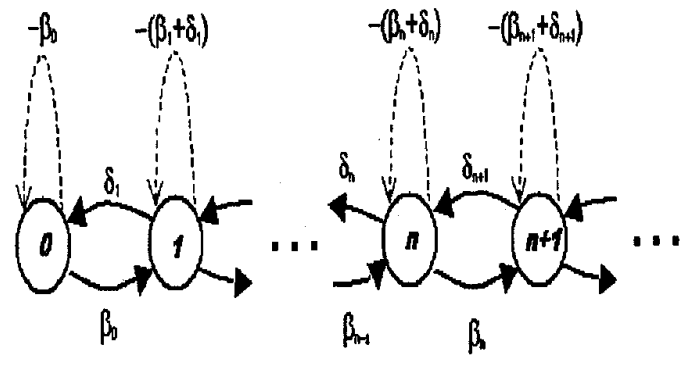

Figure 2: The Markov chain for a typical birth-death process with corresponding birth and death rates $\beta_{k}$ and $\delta_{k}$, respectively.

The assumed distribution functions are typical in the vast literature on point processes and renewal processes. They represent in some sense the most random scenario when large populations are interacting with limited resources [10]. The Markovian assumption describes the higher order statistics of $t_{i}$ and $d_{i}$, and is somewhat heuristically justified by the fact that the set of all possible radiation sources (radio transmitters, radar stations, microwave communication antennas, etc.) is usually not coordinated/correlated in any long term fashion. So for the purposes of near term prediction of $\mathbf{N}(t)$, the present knowledge about the number of active disturbances is as good as a complete history of the clisturbance activity. But the precise way in which such a prediction can be made is not fixed. For example, let $\beta_{k}(k \geq 0)$ and $\delta_{k}(k>0)$ be the Markovian transition probability rates defined by

$$
\begin{aligned}
& P\{\mathbf{N}(t+\Delta t)=k+1 \mid \mathbf{N}(t)=k\} \quad \approx \beta_{k} \Delta t \\
& P\{\mathbf{N}(t+\Delta t)=k-1 \mid \mathbf{N}(t)=k\} \approx \delta_{k} \Delta t
\end{aligned}
$$

These so called birth and death rates determine the probability of adding or removing a disturbance in the near future given the current number of disturbances. The remaining transition probability rates are taken to be zerc so that the set of all transition rates can be represented by a tridiagonal matrix $\Lambda$ (see Figure 2). Now from (A1), it follows directly that $\beta_{k}=\lambda$ for all $k \geq 0$. But the death rate, for example, could either be fixed and independent of $k$ $\left(\delta_{k}=\delta\right)$, in which case the number of current disturbances does not affect the probability that another will be removed in the near future, or the death rate might be proportional to $k\left(\delta_{k}=k \delta\right)$, then a disturbance is more likely to be removed in the near future when $k$ is large. The first scenario may seem more heuristically appealing for our application, but we will conjecture shortly that the second may agree better with the observed phenomenon. The following theorem from queuing theory is well known and applicable here [1].

Proposition 2.1 The random process $\mathbf{N}(t)$, where $t \in \mathbb{R}^{+}$, is equivalent to the state of a $(M|M| 1)$ queue if $\beta_{k}=\lambda$ for $k \geq 0$ and $\delta_{k}=\mu+\lambda$ for $k>0$ and equivalent to the state of $a(M|M| \infty)$ queue if $\beta_{k}=\lambda$ and $\delta_{k}=k \mu$ for all $k \geq 0$.

This proposition asserts that $\mathbf{N}(t)$ is stochastically equivalent to a queue with either one server or an infinite number of servers, depending on the way the higher order statistics of $\mathbf{d}_{i}$ are specified through the death rate parameters. It should be noted that in queuing theory, the second ' $M$ ' in the Kendall notation $(M|M| n)$ corresponds to exponentially distributed services times rather than total system time (or durations $d_{i}$ as specified in (A2)). These are distinct concepts when $n$ is finite. When $n=1$ it can be shown that if the arrival times and durations are as in (A1) and (A2), then the defacto service times will be exponentially distributed with parameter $\mu+\lambda$ [10]. It is also equally well known that the equilibrium state probabilities $\left\{p_{k}: k \geq 0\right\}$ of a $(M|M| 1)$ queue are geometrically distributed, while for a $(M|M| \infty)$ queue they are Poisson distributed (see Table 1). In our case, the $(M|M| 1)$ is also ergodic since $\rho<1$, while the $(M|M| \infty)$ queue is known to always be ergodic [1].

In order to relate the exosystem process, $\mathbf{N}(t)$ to the Markovian model in [8], define the (state) events disturbance absent and disturbance exists, respectively, as

$$
\begin{aligned}
& A:=\{\mathrm{N}(t)=0\} \\
& E:=\{\mathrm{N}(t)>0\}
\end{aligned}
$$

and the corresponding transition events

$$
\begin{aligned}
& A \mapsto E:=\{\mathbf{N}(t+\Delta t)>0 \mid \mathbf{N}(t)=0\} \\
& E \mapsto A:=\{\mathbf{N}(t+\Delta t)=0 \mid \mathbf{N}(t)>0\}
\end{aligned}
$$

The equilibrium state probabilities are trivially determined and shown in Table 1 . The transition probability rates can be shown to be:

$$
\begin{aligned}
& \Lambda_{A E}=\beta_{0} \\
& \Lambda_{E A}=\delta_{1} \frac{p_{1}}{1-p_{0}}
\end{aligned}
$$

which reduce to the expressions given in Table 2 for the specific processes we are considering. Now in the event that disturbances are rare, we can assume that the disturbance arrival density $\lambda$ is small relative to the average duration of a disturbance $\mu$. We denote the corresponding state and transition probability rates with either a superscript or subscript $r$. In the case of the $(M|M| 1)$ queue it turns out that the rare event assumption does not provide any addition simplification (approximations), but it does clarify the $(M|M| \infty)$ case a bit. As can be seen in Tables 1 and 2 , the two queue models are virtually identical for rare events, but as $\lambda$ increases the $(M|M| \infty)$ queue is less likely to be in the $E$ state, as is expected. A comparison of these results to those in [8] reveals that their exosystem model (the non 'burst model' 


\begin{tabular}{|c|c|c|}
\hline & $(M|M| 1)$ & $(M|M| \infty)$ \\
\hline \hline$\rho$ & $\frac{\lambda}{\mu+\lambda}$ & $\frac{\lambda}{\mu}$ \\
\hline$p_{k}$ & $(1-\rho) \rho^{k}$ & $e^{-\rho} \frac{\rho^{k}}{k !}$ \\
\hline$P(A)$ & $1-\rho$ & $e^{-\rho}$ \\
\hline$P(E)$ & $\rho$ & $1-e^{-\rho}$ \\
\hline$P(A)_{r}$ & $1-\rho$ & $1-\rho$ \\
\hline$P(E)_{r}$ & $\rho$ & $\rho$ \\
\hline
\end{tabular}

Table 1: Equilibrium state probabilities associated with the single-server and infinite-server queues.

\begin{tabular}{|c|c|c|}
\hline & $(M|M| 1)$ & $(M|M| \infty)$ \\
\hline \hline$\Lambda_{k, k+1}$ & $\lambda$ & $\lambda$ \\
\hline$\Lambda_{k, k-1}$ & $\mu+\lambda$ & $k \mu$ \\
\hline$\Lambda_{A E}$ & $\lambda$ & $\lambda$ \\
\hline$\Lambda_{E A}$ & $\mu$ & $\lambda \frac{e^{-p}}{1-e^{-p}}$ \\
\hline$\Lambda_{A E}^{\prime}$ & $\lambda$ & $\lambda$ \\
\hline$\Lambda_{E A}^{*}$ & $\mu$ & $\mu-\lambda$ \\
\hline
\end{tabular}

Table 2: Transition probability rates associated with the single-server and infinite-server queues.

variety) is an $(M|M| 1)$ queue. The problem in using such a model is that the notion of service time in our application has no obvious interpretation or relevance. The disturbances applied to a flight controller are not being serviced by the computer, and thus system time and service time should be synonymous. It is for this reason that we conjecture that the statistics of $\mathbf{N}(t)$ are more likely to be equivalent to those of a $(M|M| \infty)$ queue, where indeed service time and system time are always equivalent.

\section{The Interference Model}

We assume the aircraft in a specific flight regime can be modeled by the sampled-data system

$$
\begin{aligned}
x(i+1) & =A x(i)+B u(i) \\
y(i) & =C x(i),
\end{aligned}
$$

where $A \in \mathbb{R}^{\mathrm{n} \times \mathrm{n}}, B \in \mathbb{R}^{\mathrm{n} \times \mathrm{m}}$ and $C \in \mathbb{R}^{\mathrm{p} \times \mathrm{n}}$. When no radiation is present, the nominal closed-loop system with $u=r-F x$ will be denoted by $\left(A_{0}, B, C\right)$. Now define the interference mapping as

$$
\begin{aligned}
\mathcal{I}: & Z^{+} \mapsto \mathbb{R}^{\mathrm{m} \times \mathbf{n}} \times \mathbb{R}^{\mathbf{m} \times \mathbf{m}} \times[0,1] \\
: & k \mapsto\left(\Delta F_{k}, G_{k}, p_{k}^{*}\right),
\end{aligned}
$$

where $Z^{+}:=\{0,1,2, \ldots\}$ and $\Delta F_{k}$ denotes the perturbation to the nominal state space gain matrix applied with probability $p_{k}^{*}$ when $\mathbf{N}(t)=k$. The ma$\operatorname{trix} G_{k}$ is a weighting of the disturbance sequence $\left\{\mathbf{r}_{d}(i): i \in Z^{+}\right\}$that is injected into the closed-loop also with probability $p_{k}^{*}$. (For our linear plant model, closed-loop stability will not be affected by this noise injection.) Let $\theta(i)$ denote the sampled exosystem state $\mathbf{N}(t)$, i.e., $\theta(i)=\mathbf{N}(i T)$, where $T$ is the sampling period. If $T$ is small then this discrete-time Markov process can be characterized by the transition probability matrix $P=e^{\Lambda T} \approx I+\Lambda T$. Now when $\mathbf{N}(t)=k$, the probability of an upset condition is $p_{k}^{*}$. Thus we can define a second discrete-time Markov process $\left\{\tilde{\theta}(i): i \in Z^{+}\right\}$consisting of two states for each state of $\left\{\theta(i): i \in Z^{+}\right\}$. We use the convention that

$$
\tilde{\theta}(i)=\left\{\begin{array}{cll}
\text { even } \ell \in Z^{+} & : & \text {no upset and } \theta(i)=\frac{\ell}{2} \\
\text { odd } \ell \in Z^{+} & : & \text {upset and } \theta(i)=\frac{\ell-1}{2}
\end{array} .\right.
$$

It can be easily verified that the corresponding transition probability matrix is

$$
\tilde{P}=\left(P \otimes \amalg_{2}\right) \cdot \operatorname{diag}\left(1-p_{0}^{*}, p_{0}^{*}, 1-p_{1}^{*}, p_{1}, \ldots\right),
$$

where $\otimes$ denotes the Kronecker product and $\amalg_{2} \in$ $\mathbb{R}^{2 \times 2}$ is a matrix with a one in every entry. With this setup, the closed-loop input assumes the form

$$
\mathbf{u}(i)=r(i)-\left(F+\Delta F_{\tilde{\theta}(i)}\right) \mathbf{x}(i)+G_{\tilde{\theta}(i)} \mathbf{r}_{d}(i)
$$

yielding a family of closed-loop systems

$$
\begin{aligned}
\mathbf{x}(i+1) & =A_{\tilde{\theta}(i)} \mathbf{x}(i)+B_{\tilde{\theta}(i)} \mathbf{r}_{d}(i)+B r(i) \\
\mathbf{y}(i) & =C \mathbf{x}(i),
\end{aligned}
$$

where for $\ell \in Z^{+}$:

$$
\begin{aligned}
& A_{\ell}=\left\{\begin{array}{cl}
A-B F & : \quad \ell \text { even } \\
A-B\left(F+\Delta F_{\ell}\right) & : \quad \ell \text { odd }
\end{array}\right. \\
& B_{\ell}=\left\{\begin{array}{ccc}
0 & : & \ell \text { even } \\
B G_{\ell} & : & \ell \text { odd. }
\end{array}\right.
\end{aligned}
$$

The following stability definition and related theorems for this particular class of systems have been adapted from [4] assuming that:

(A4) $\tilde{\theta}(i) \approx \hat{\theta}(i)$, a finite state Markov chain with $\bar{\ell}$ states, transition probabilities $\hat{P}$ and initial distribution $\nu$.

(A5) The initial state $\mathbf{x}(0)=\mathbf{x}_{0}$ is a second-order random variable.

(A6) $\left\{\mathbf{r}_{d}(i): i \in Z^{+}\right\}$is a second-order independent wide sense stationary sequence of random variables with mean $r$ and covariance matrix $R$.

(A7) The random variables $\mathbf{x}_{0}$ and $\left\{\hat{\theta}(i): i \in Z^{+}\right\}$ are independent of $\left\{\mathbf{r}_{d}(i): i \in Z^{+}\right\}$.

The approximation in (A4) is in the following sense: if the equilibrium probability of having $\mathbf{N}(t)>\bar{k}$ is small for some $\bar{k}>0$ then truncating the state space to the first $\bar{k}$ states, including the zero state, yields approximately the same model stochastically. Note $\hat{P}$ is equivalent to the upper left $(2 \bar{l} \times 2 \bar{l})$ submatrix of $\tilde{P}$ where $\bar{\ell}=2 \bar{k}$. 
Definition 2.1 The system (3) with $r(t) \equiv 0$ is mean square stable if for any initial condition $\mathbf{x}_{0}$, initial distribution $\nu$, and input disturbance $\left\{\mathbf{r}_{d}(i)\right.$ : $\left.i \in Z^{+}\right\}$satisfying $(A 4)-(A 7)$ there exists a matrix $Q \in \mathbb{R}^{\mathbf{n} \times \mathbf{n}}$ not depending on $\mathbf{x}_{o}$ such that

$$
\|Q(i)-Q\| \rightarrow 0 \text { as } i \rightarrow \infty,
$$

where $Q(i):=E\left(\mathbf{x}(i) \mathbf{x}^{T}(i)\right)$.

Theorem 2.1 If the system (3) with $r(t) \equiv 0$ is mean square stable then there exists a vector $q \in \mathbb{R}^{\mathbf{n}}$ such that

$$
\|q(i)-q\| \rightarrow 0 \text { as } i \rightarrow \infty,
$$

where $q(i):=E(\mathbf{x}(i))$.

Theorem 2.2 The homogenous version of system (3)

$$
\mathbf{x}(i+1)=A_{\hat{\theta}(i)} \mathbf{x}(i)
$$

is almost surely convergent to 0 , i.e., $\mathbf{x}(i) \rightarrow 0$ w.p. 1 as $i \rightarrow \infty$.

Theorem 2.3 The system (3) with $r(t) \equiv 0$ is meansquare stable if and only if the spectral radius of

$$
\mathcal{A}_{1}:=\left(\hat{P}^{T} \otimes I_{n^{2}}\right) \operatorname{diag}\left(A_{0} \otimes A_{0}, A_{1} \otimes A_{1}, \ldots, A_{\overline{\ell-1}} \otimes A_{\overline{\ell-1}}\right)
$$

is strictly less than one. $\left(I_{n^{2}}\right.$ is the $n^{2} \times n^{2}$ identity matrix.)

These results clearly illustrate the advantages of using the mean square stability concept. It is easy to test for, it implies stability of the mean dynamics, and it yields almost sure asymptotic stability of the zeroinput state space trajectories. These ideas are further illustrated in the following simple example.

Example 2.1 We consider the rare event scenario discussed in the previous section, where $\lambda$ is taken to be small relative to $\mu$. According to Table $1, p_{k} \approx 0$ for $k>1$, and thus the finite state Markov chain $\hat{\theta}(i)$ has $\bar{\ell}=2 \bar{k}=4$ states. In Kronecker notation, the corresponding transition probability matrix is

$$
\begin{gathered}
\hat{P}=\left(\left[\begin{array}{cc}
1-\lambda T & \lambda T \\
\mu T & 1-\mu T
\end{array}\right] \otimes\left[\begin{array}{cc}
1 & 1 \\
1 & 1
\end{array}\right]\right) \\
\operatorname{diag}\left(\mathrm{l}-p_{0}^{*}, p_{0}^{*}, 1-p_{1}^{*}, p_{1}^{*}\right) .
\end{gathered}
$$

We next make the sirnplifying assumptions that $p_{0}^{*}=$ $0, p_{1}^{*}=1$, and $A_{0}=A_{1}=A_{2}$, so that

$$
\mathcal{A}_{1}=\left[\begin{array}{cc}
(1-\lambda T)\left(A_{0} \otimes A_{0}\right) & (1-\lambda T)\left(A_{0} \otimes A_{0}\right) \\
0 & 0 \\
0 & 0 \\
\lambda T\left(A_{0} \otimes A_{0}\right) & \lambda T\left(A_{0} \otimes A_{0}\right) \\
\mu T\left(A_{0} \otimes A_{0}\right) & \mu T\left(A_{3} \otimes A_{3}\right) \\
0 & 0 \\
0 & 0 \\
(1-\mu T)\left(A_{0} \otimes A_{0}\right) & (1-\mu T)\left(A_{3} \otimes A_{3}\right)
\end{array}\right] .
$$

Now consider the first order case, $n=1$, where $a_{0}:=$ $A_{0}$ and $a_{3}:=A_{3}$. A direct calculation of the spectrum of $\mathcal{A}_{1}$ (again assuming that $T^{2} \approx 0$ ) gives

$$
\sigma\left(\mathcal{A}_{1}\right)=\left\{0,0,(1-\lambda T) a_{0}^{2},(1-\mu T) a_{3}^{2}\right\} .
$$

Hence the spectral radius of $\mathcal{A}_{1}$ is

$$
r_{\sigma}\left(\mathcal{A}_{1}\right)=\max \left\{(1-\lambda T) a_{0}^{2},(1-\mu T) a_{3}^{2}\right\} .
$$

Since by design we normally have $(1-\lambda T) a_{0}^{2} \approx a_{0}^{2}<$ 1 , it follows from Theorem 2.3 then that the closedloop system will be mean square stable if and only if

$$
(1-\mu T) a_{3}^{2}<1
$$

or equivalently,

$$
P(E \mapsto E) \approx 1-\mu T<\frac{1}{a_{3}^{2}}
$$

(Here we have defined the event $E \mapsto E=\{\mathbf{N}(t+$ $T)>0 \mid \mathbf{N}(t)>0\}$.) If $a_{3}$ is stable then there is no upperbound on the persistence of the radiation from sample to sample. But in the case of an unstable perturbation, there is clearly an inverse relationship between how unstable the perturbed system can be and the average duration of rare radiation events. Such a relation could obviously be exploited to robustify the stability of the closed loop system to this type of phenomenon.

In an experimental setting like the one that exists at the NASA Langley Research Center, the exosystem can be completely controlled by the test chamber operator. That is, the event arrivals, durations, and intensities are routinely preprogrammed into a test run. As mentioned in the introduction, some statistical characterization of radiation events in the atmosphere exists in the literature, though not quite in the direct form required for parameterizing our exosystem model. For example, one may think of radiation intensity (as measure by the field strength) to be some measure of the number of active sources (i.e, $\mathbf{N}(\mathrm{t})$ ), but in practice the intensity is more continuous in nature, so that our queue states act as a quantization of the actual phenomenon. However, in a controlled laboratory environment, discrete radiation levels are easily programmed. So from the point of view of the exosystem model development, the real open question is how to take existing environmental data (or create new data) and map it over to some realistic laboratory test configuration. Of course, an advantage of the laboratory setting is that severe radiation environments can also be created to test the upperbound on the electromagnetic susceptibility of a given component even though such environments are not likely to be found in nature.

Parameterizing the interference model is a considerably more difficult problem. This is mainly due to the fact that getting direct access to internal computer data, frame by frame, and computing control law perturbation statistics is a very formidable task. 
At best, current test fixtures can only record inputoutput data from the flight controller, and the volume of data stored is virtually unmanageable. For this reason, we have steered away from introducing stochastic perturbation models, as done in [8]. What we envision instead is a kind of worst case deterministic analysis of how much control law perturbation can be tolerated given a particular radiation environment before instability sets in. Our stability margins will be conservative, but they do not required us to guess about the statistical nature of unobserved internal data perturbations.

\section{Conclusions and Future Research}

In this paper we developed a mathematical model which can be used for stability analysis and augmentation of a digitally implemented control law. The model was composed of a Markovian exosystem supplying radiation events to a discrete-time jump linear system which models how the radiation interferes with the nominal operation of the closed-loop system. It was proposed that the exosystem model is equivalent to an $(M|M| \infty)$ queue with appropriately set transition probability rates. The interference model mapped each queue state to an appropriate deterministic perturbation of the control law which was introduced with a certain probability, also a function of the queue state. (There was also a corresponding noise injection system, but for linear plant models, closed-loop stability is not affected by additive noise.) It was then demonstrated by example that the notion of mean square stability could be used in conjunction with this model to develop a relationship between stability bounds on the perturbed system and characteristics of the radiation. Much future work remains to be done. At present, simulation platforms and experimental testbeds are being developed to put this theory to the test. Furthermore, the simple example from the previous section is being generalized to give systematic stability robustness measures for more realistic systems. This work will be reported in future publications.

\section{Acknowledgement}

This research was supported by the NASA Langley Research Center under contract number NAS1-19858108.

\section{References}

[1] S. Asmussen, Applied Probability and Queues, Chichester: Wiley Press, 1987.

[2] C. M. Belcastro, 'A Low-Cost Comprehensive Process for Assessing Electromagnetic Environment (EME) Effects on Flight-Critical Control Computers,' Proc. 15th DASC Digital Avionics Systems Conference, New York, NY, 1996, pp. 443-449.

[3] B. T. Clough, 'Effects of Electromagnetic Interference on Digital Control Systems,'Internal Report Number WL-TR-96-3122, Wright Laboratory, Wright-Patterson AFB, OH, 1996.
[4] O. L. V. Costa and M. D. Fragoso, 'Stability Results for Discrete-Time Linear Systems with Markovian Jumping Parameters,' Journal of Mathematical Analysis and Applications, vol. 179,1993 , pp. 154-178.

[5] Federal Aviation Administration, 'Certification of Aircraft Electrical/Electronic Systems for Operation in the High Intensity Radiated Fields (HIRF) Environment,' Proposed FAA Advisory Circular ARD 50040, Draft 16, August, 1993.

[6] P. M. Frank, 'Fault Diagnosis in Dynamic Systems Using Analytical and Knowledge-Based Redundancy: A Survey and Some New Results,' Automatica, vol. 26, no. 3, 1990, pp. 459-474.

[7] R. Isermann, 'Process Fault Detection Based on Modeling and Estimation Methods - A Survey,' Automatica, vol. 20, no. 4, pp. 1984, pp. 387-404.

[8] H. Kim, A. L. White and K. G. Shin, 'Susceptibility of Control Computers to Environmental Disruptions and Its Effects on System Stability,' Proc. 1996 IEEE Aerospace Applications Conference, Aspen, CO, 1996, pp. 309-320.

[9] G. Pan and Y. Bar-Shalom, 'Stabilization of Jump Linear Gaussian Systems Without Mode Observations, Inter. Journal of Control, vol. 64, no. 4,1996 , pp. $631-661$.

[10] A. Papoulis, Probability, Random Variables, and Stochastic Processes, 3rd Edition, New York: McGraw-Hill, 1991.

[11] M. L. Shooman, 'A Study of Occurrence Rates of Electromagnetic Interference (EMI) to Aircraft with a Focus on HIRF (external) High Intensity Radiated Fields,' NASA Report CR-194895, April 1994.

[12] R. Srichander and B. K. Walker, 'Stochastic Stability Analysis for Continuous-Time Fault Tolerant Control Systems,' Inter. Journal of Control, vol. 57, no. 2,1993 , pp. $433-452$.

[13] D. A. Sworder, 'Control of Systems Subject to Sudden Change in Character,' Proc. IEEE, vol. 64, no. 8,1976 , pp. $1219-1225$.

[14] A. L. White and H. Kim, 'Designing Experiments for Controller Perturbation Theories - An Example,' Proc. 1996 IEEE Aerospace Applications Conference, Aspen, CO, 1996, pp. 265-278.

[15] A.S. Willsky, 'A Survey of Design Methods for Failure Detection in Dynamic Systems,' Automatica, vol. 12 , no. 6,1976 , pp. 601-611.

[16] Y. C. Yeh, 'Triple-Triple Redundant 777 Primary Flight Computer,' Proc. 1996 IEEE Aerospace Applications Conference, Aspen, CO, 1996, pp. 293-307. 